\title{
Evaluation of a direct colorimetric assay for rapid detection of rifampicin resistant Mycobacterium tuberculosis
}

\author{
Dawit WoldeMeskel ${ }^{1}$, Getahun Abate ${ }^{1}$, Mekuria Lakew², Solomon Goshu ${ }^{3}$, Alemayehu Selassie ${ }^{3}$, Hakan \\ Miorner ${ }^{4}$, Abraham Aseffa ${ }^{1}$
} \begin{abstract}
bromide (MTT) for a rapid detection of rifampicin resistance. results were already available by the end of week two. [Ethiop.J.Health Dev. 2005;19(1):51-54]

Introduction

Early detection of drug resistance is one of the essential steps in the management of tuberculosis. The effectiveness of a standard anti-TB treatment regimen correlates well with the in-vitro drug susceptibility pattern of the infecting tubercle bacilli. The results of drug susceptibility tests help select a proper treatment regimen or modify treatment regimen for a better management of patients and for surveillance and timely control of the spread of drug- resistant $\mathrm{TB}$ in the community.
\end{abstract}

Background: Susceptibility test is a useful tool for selecting and modifying appropriate treatment for tuberculosis. Objective: To evaluate a direct colorimetric assay based on 3-(4,5-dimethylthiazol-2-yl)- 2,5-diphenyl tetrazolium

Methods: Sputum was inoculated directly into 7H9 broth supplemented with PANTA (antibiotic mixture) and oleic acid albumin dextrose catalase (OADC) to which MTT was added after 1-3 weeks. Bacterial growth is indicated by the formation of a colored formazan product, which can be measured with a spectrophotometer. The assay was read on the first, second or third week of incubation depending on when a measurable formazan was observed in the drug free control tube. The results were compared against the standard indirect modified proportion method of susceptibility testing on 7H10 media. Single sputum samples from 78 re-treatment cases were tested.

Results: The MTT assay was found to be $90.5 \%$ sensitive and $100 \%$ specific. In more than two-thirds of the samples,

Conclusion: The direct colorimetric assay is a simple rapid test for rifampicin susceptibility testing. It significantly shortens the time required to obtain a drug susceptibility test result and could be useful to screen for MDR-TB.

The drug susceptibility testing of $\mathrm{M}$. tuberculosis can be done either indirectly using cultured mycobacterial colonies isolated from clinical specimens or directly on the clinical samples (1). Conventional susceptibility testing is done after bacterial isolation using agar-based or egg-based media and takes seven to twelve weeks. It is time consuming and laborious. The direct susceptibility test with conventional methods takes three to four weeks. The time required for the susceptibility test is significantly reduced when relatively new methods like a standard radiometric method (BACTEC) are used. In the BACTEC method, the direct and indirect tests need an average time of 11 and 18 days respectively (2). However, BACTEC is very expensive for routine use in resource-poor settings.
We had previously developed an affordable and reliable colorimetric assay using a dye called 3-[4, 5dimethylthiazol-2-yl]-2, 5- diphenyl tetrazolium bromide (MTT) for a rapid detection of rifampicin resistance in M. tuberculosis isolates using the BACTEC method as a reference (3). A colorimetric assay using MTT was first introduced by Mossman as a quantitative measure of mammalian cell survival and proliferation (4). MTT is a yellow tetrazolium salt, which is converted into a blue formazan by dehydrogenases of a live cell. The assay is based on the principle that the amount of formazan produced is directly proportional to the number of live cells (4). The formazan can then be measured by a spectrophotometer after solubilization (5).

We previously reported on the indirect MTT assay in which susceptibility testing is done on a mycobacterial isolate. The MTT-based antimycobacterial drug susceptibility test requires only three to seven days $(3,6)$. However, the time required to obtain a primary isolate to perform an indirect susceptibility test is relatively longer and may limit the wider application of new rapid methods.

In this study, we evaluated a direct MTT assay for rapid detection of rifampicin-resistant $M$. tuberculosis in sputum samples collected under program conditions.

\footnotetext{
${ }^{1}$ Armauer Hansen Research Institute, P.O.Box 1005, Tel. 2511 211375, Fax 2511 211653, E-mail: ahridir@telecom.net.et, aseffaa@yahoo.com, Addis Ababa, Ethiopia; ${ }^{2}$ Department of Biology, Addis Ababa University; ${ }^{3}$ St Peter TB Specialized Hospital, Addis Ababa; ${ }^{4}$ Department of Medical Microbiology, Dermatology and Infection, Lund University, Lund, Sweden
} 


\section{Methods}

Specimens

Sputum samples were collected from 100 consecutive, consenting re-treatment cases (patients who became smear positive again after a previous treatment for tuberculosis) visiting St Peter TB Specialized Hospital in Addis Ababa between December 2001 and October 2002. Sputum specimens were collected in screw-capped clean plastic sputum cups and kept at $4{ }^{\circ} \mathrm{C}$ until transported within four hours of collection to the TB laboratory at the Armauer Hansen Research Institute (AHRI) where they were processed immediately.

\section{Culture}

The sputum was digested and decontaminated using Petroff's method (7). The pellets were resuspended in 2.5 $\mathrm{ml}$ PBS. An aliquot of $100 \mu \mathrm{l}$ of the sample was then cultured into two tubes containing Lowenstein Jensen (LJ) media for primary isolation. The tubes were incubated at $37^{\circ} \mathrm{C}$ and examined weekly for bacterial growth until the eighth week. An aliquot of $500 \mu l$ from the remaining resuspended sample was inoculated into each of four tubes containing 3ml Middlebrook 7H9 broth supplemented with Oleic acid Albumin Dextrose Catalase (OADC) enrichment (Becton-Dickinson) and antibiotics mixture (PANTA) (Becton-Dickinson). PANTA is a standard antibiotic cocktail that contains Polymyxin B, Amphotericin B, Nalidixic acid, Trimethoprim and Azlocillin. One of the four tubes contained rifampicin at $2 \mathrm{mg} / \mathrm{l}$ final concentration (Sigma) while the rest were drug free controls. They were then incubated at $37^{\circ} \mathrm{C}$ and the MTT assay was done on the day after a visible formazan product was observed in the control medium. However, when no formazan was seen on the first and second week of incubation, the assay would be performed in the third week.

\section{MTT assay}

The MTT assay was done as described previously (8). Briefly, $300 \mu \mathrm{l}$ of $5 \mathrm{mg} / \mathrm{ml}$ MTT solution was added into each tube. The tubes were vortexed and incubated for 4 hrs at $37^{\circ} \mathrm{C}$. The formazan produced was dissolved with solubilization buffer ( $20 \%$ sodium dodecyl sulfate) in a $50 \%$ aqueous solution of dimethyl formamide Optical density $\left(\mathrm{OD}_{570}\right)$ was then measured at $570 \mathrm{~nm}$ (Novaspec II photometer, Pharmacia Biotech Ltd, UK), using a tube containing 7H9 broth, PBS, MTT and solubilization buffer as a reference. Relative optical density unit (RODU) values were calculated by dividing the OD of the drug containing tubes with the OD of drug free control.

The correlation of the MTT assay at $\mathrm{OD}_{570}$ in $7 \mathrm{H} 9$ broth to colony forming units on standard 7H10 agar media was determined in initial experiments. Based on the distinctive difference between positive and negative samples observed, appropriate cut-off values were determined. No CFU was observed for broth cultures with $<0.1 \mathrm{OD}_{570}$. Therefore, the MTT results were defined as interpretable if OD of a control tube was $\geq 0.1$. A strain was defined as rifampicin-susceptible when the relative optical density unit (RODU) value was below 0.2 and resistant when it was above 0.5 . RODU between 0.2 and 0.5 was considered indicative of borderline resistance.

Among the four tubes, two control tubes were used to check formazan formation on the first and second weeks. The MTT assay on the rifampicin containing tube and one control was performed a day after MTT assay on a control tube showed visible formazan formation. When the MTT assay on control tubes did not show formazan formation in the first two weeks, the remaining tubes were used on the third week.

A test for bacterial contamination was performed for each sample inoculated into 7H9 broth by inoculation of a loopful of the corresponding broth on nutrient agar and incubating it at $37^{\circ} \mathrm{C}$ for 24 hours before performing the MTT assay on the particular tube. Mycobacterial isolates were identified as $M$. tuberculosis using standard biochemical tests (9).

\section{Results}

Of 100 processed samples, 78 gave interpretable results (Table 1). The rest were excluded because of contamination of media $(n=4)$, lack of growth in drugfree control medium of MTT assay (OD $\left.{ }_{570}<0.1\right)(n=7)$ and lack of growth on LJ medium $(n=11)$. From the 11 isolates that were culture negative five grew on 7H9 broth and had MTT results. Among the five isolates, four were susceptible to rifampicin and one was resistant. Three of the culture negative isolates came out with uninterpretable MTT result. Among the seven isolates that showed uninterpretable MTT result only two did not show growth on LJ while the others had growth and most were MDR. A relative value, RODU was employed to control for possible strain differences in the ability to reduce MTT (8) and define resistance and susceptibility. The mean ( \pm standard deviation, SD) RODU value of resistant strains was $1.08 \pm 0.5$ whereas the mean $( \pm \mathrm{SD})$ RODU value of susceptible strains was $0.04 \pm 0.06$. Except for one susceptible isolate with an RODU of 0.3, all others had an RODU value $<0.2$. This result matched with the RODU values for resistant and susceptible strains in the standardized indirect MTT assay for rifampicin reported earlier $(3,6,8)$. 
Table 1: Comparison of the sensitivity test results using direct MTT assay and the standard proportional method

\begin{tabular}{lllll}
\hline \multirow{2}{*}{ MTT assay } & \multicolumn{3}{l}{ Standard proportional method } & Total \\
\cline { 2 - 5 } & Susceptible & Intermediate & Resistant & 58 \\
\hline Susceptible & 56 & 0 & 2 & 1 \\
Intermediate & 1 & 0 & 0 & 19 \\
Resistant & 0 & 0 & 19 & 78 \\
\hline Total & 57 & 0 & 21 & \\
\hline
\end{tabular}

Of 100 original samples, 22 were excluded from analysis because of contamination of media $(n=4), O D_{570}$ below 0.1 in drug-free control tubes of MTT assay $(n=7)$ and due to no growth on LJ media $(n=11)$.

Out of the 78 isolates tested for rifampicin resistance the MTT assay identified $19(24.4 \%)$ as resistant and 59 (75.6\%) as susceptible. The results matched with those obtained using the gold standard method of susceptibility testing except for 2/78 samples which were discordant, reported as susceptible by the MTT assay but were resistant according to the proportional method (Table 1). The MTT assay had thus a sensitivity of $90.5 \%$, specificity of $100 \%$ and positive and negative predictive values of $100 \%$ and $96.6 \%$, respectively.

The time required to obtain susceptibility test results was shorter for the MTT assay. Among the 78 samples analysed in this study, 15 (19.2\%) were interpreted at week one, another 38 a week later, making a total of 53 $(67.9 \%)$ by week two, and all $78(100 \%)$ by week three. Among the 19 rifampicin resistant isolates, one gave interpretable result during the first week of incubation, 10 of them during the second, and 8 during the third weeks. All rifampicin susceptible and rifampicin resistant samples were correctly identified with the naked eye, suggesting that visual reading could be sufficient to interpret the assay.

\section{Discussion}

Early diagnosis of infection with multi-drug resistant strains of $M$. tuberculosis is important for individual case management as well as for control of the spread of MDRTB in a particular area. In vitro susceptibility tests on mycobacteria are helpful to clinicians in selecting the potentially most effective anti-mycobacterial agent. These tests can be either direct or indirect. In the direct test, smear positive sputum is directly inoculated onto drug-containing (test) and drug-free (control) media. The indirect test requires primary isolation of the organisms (6).

This study demonstrates that a simple rapid colorimetric assay can be used to determine the susceptibility or resistance of the organisms to rifampicin in a sample directly without the need to isolate the mycobacteria first.

This shortens the time required for a susceptibility reporting by three to five weeks compared to the standard method. The maximum period required to complete all the investigations with the MTT assay for the 78 specimens analysed in this study was three weeks.
Our results of the MTT assay were confirmed by the standard proportion method on Middlebrook 7H10 using strains isolated on LJ media from the same samples. The result matches $97.3 \%$ with drug susceptibility result of the proportion method. The assay did not correctly determine resistance to rifampicin in two samples. A similar study done in the same laboratory on samples from other smear positive pulmonary TB patients had $100 \%$ agreement with the proportional method (8). The difference in the protocols of the two direct MTT assays was that the latter utilized three separate culture tubes for drug-free $7 \mathrm{H} 9$ broth control instead of just one as in this study. A total of four tubes (three controls and one rifampicin containing tube) were used in our study. Where the single control tube fails to yield comparable bacterial growth and give appropriate OD value, the calculated RODU may be mistakenly low. This might lead to resistance being missed. Although the sensitivity and specificity of the assay is still high, it is evident that the type of error has to be minimized by increasing the number of control tubes to three, to be able to use one control tube per week.

The rate of MDR TB in Ethiopia is on the rise. Determination of resistance against rifampicin in sputum samples from smear positive TB patients using a simple rapid colorimetric assay is a useful tool for individual patient management as well as for monitoring MDR TB in a control programme. The direct MTT assay reported in this study is proposed as an alternative drug susceptibility testing method in the country. Since the performance of any test may vary according to prevalence, further evaluation of the direct MTT assay under different rates of drug resistance is recommended.

\section{Acknowledgement}

This project was supported by a grant from UNDP/ WORLD BANK/ WHO Special Programme for Research and Training in Tropical Diseases (TDR). This study was part of the MSc thesis of DW at Addis Ababa University.

\section{References}

1. Canetti G, Froman S, Grosset J et al. Mycobacteria: Laboratory methods for testing drug sensitivity and resistance. Bull World Health Org 1963; 29: 565 575. 
2. Heifets LB. Drug susceptibility tests in the management of chemotherapy of tuberculosis. In: Heifets LB, ed. Drug susceptibility in the chemotherapy of mycobacterial infections. CRC Press, Inc. USA, 1991: 89- 120.

3. Mshana RN, Genet T, Getahun A, Hakan M. Use of 3-[4, 5-dimethylthiazol-2-yl]-2, 5- diphenyl tetrazolium bromide for rapid detection of resistant M. tuberculosis. J Clin Microbiol 1998; 36: 12141219.

4. Mosmann T. Rapid colorimetric assay for cellular growth and survival: application to proliferation and cytotoxicity assays. J Immunol Methods 1983; 65: 624.

5. Denizot F, Lang R. Rapid colorimetric assay for cell growth and survival. Modifications to the tetrazolium dye procedure giving improved sensitivity and reliability. J Immunol Methods 1986;89:271.
6. Abate G, Mioner H, Ahmed D, Hoffner Se. Drug resistance in $M$. tuberculosis strains isolated from retreatment cases of pulmonary tuberculosis in Ethipioa: susceptibitlity to first line and alternative druges. Int J Tuberc Lung Dis 1998;2:580-584.

7. Petroff SA. A new and rapid method for the isolation and cultivation of tubercle bacilli directly from the sputum and feces. J Exp Med 1915; 21: 38-42.

8. Abate G, Aseffa A, Sellassie A, et al. Direct colorimetric assay for rapid detection of rifampicin resistant Mycobacterium tuberculosis. J Clin Microbiol 2003; 42 (2): 871-873.

9. Kent P.T, Kubica G.P. Public Health Mycobacteriology: A Guide for the Level III Laboratory. CDC, Atlanta, Georgia 30333, 1985. 
